\title{
LOOKING FOR TIPS TO FIND ICEBERGS - SURVEILLANCE OF HAEMOLYTIC URAEMIC SYNDROME TO DETECT OUTBREAKS OF SHIGA TOXIN-PRODUCING E. COLI INFECTION
}

\author{
Dirk Werber (werberd@rki.de) ${ }^{1}$, Christina Frank ${ }^{1}$, Maria Wadl ${ }^{1,2}$, Helge Karch ${ }^{3}$, Angelika Fruth ${ }^{4}$, Klaus Stark $^{1}$ \\ 1.Department for Infectious Disease Epidemiology, Robert Koch Institute, Berlin, Germany \\ 2. Postgraduate Training for Applied Epidemiology („PAE”, German Field Epidemiology Training Programme) \\ 3. Institute of Hygiene, National Consulting Laboratory on Haemolytic Uraemic Syndrome, University of Münster, Germany \\ 4. National Reference Centre for Salmonella and other Bacterial Enteric Pathogens, Robert Koch Institute, Wernigerode, Germany
}

To the Editor: In a recent article, De Schrijver et al. described an outbreak of Verocytotoxin-producing Escherichia coli, syn. Shiga toxin-producing $E$. coli (STEC), linked to the consumption of ice cream produced at a farm in the province of Antwerp, Belgium [1]. Interestingly, the outbreak was identified through the timeclustering of patients who had developed haemolytic uraemic syndrome (HUS) and from whom STEC 0145 was isolated. HUS a triad of acute renal injury, micro-angiopathic haemolytic anaemia and thrombocytopenia - predominantly affects children. A subset of STEC-infected patients (circa $5-15 \%$ in patients infected with serotype 0157:H7) develop HUS, and more than $80 \%$ of childhood HUS is attributable to STEC infection [2].

E. coli 0157:H7 dominates STEC disease statistics in many countries. Routine detection of this serotype in stool, by use of sorbitol-MacConkey (SMAC) agar, is well-established, timely and inexpensive. Other ("non-0157:H7") STEC serotypes cannot be identified on SMAC agar because these strains ferment sorbitol. Their diagnosis is more complex and requires a sequential approach that entails screening for Shiga toxin or Shiga toxin genes, followed by isolation and serotyping of STEC. However, many laboratories do not screen stool for Shiga toxin or Shiga toxin genes; in some countries, non-0157:H7 STEC are not routinely sought at all. This results in a substantial under-ascertainment of non-0157:H7 STEC. Compared to most other bacterial pathogens, even the prominent STEC 0157:H7 infections are less likely to be diagnosed, for at least two reasons: firstly, the culturing of stool on SMAC agar is underused (e.g. diagnosis is often sought only in patients with bloody diarrhoea) or not routinely performed at all (e.g. in Belgium [1]). Secondly, in countries where STEC identification is based solely on screening for Shiga toxin or Shiga toxin genes, underdiagnosis of 0157:H7 infections is likely if laboratories are paid a fixed fee, which does not cover the cost of subsequent strain isolation and serotyping (e.g. in Germany).

Identifying STEC outbreaks through a cluster of HUS patients has been a characteristic of other non-0157:H7 STEC outbreaks, e.g. a large STEC 0111 outbreak in Australia [3] and a recent STEC 0103 outbreak in Norway [4]. Both were food-borne, and the food vehicle was identified, potentially allowing food safety to improve. Notably, even within the 0157 serogroup, several outbreaks caused by the emerging sorbitol-fermenting (SF) strain of STEC 0157:Hin Germany were only detected by an increase in the number of paediatric HUS patients. Likewise, an investigation of an outbreak of SF STEC 0157:H- infection in the United Kingdom was triggered by epidemiologic investigations of paediatric HUS patients [5].
We believe that a timely, preferably active, surveillance of HUS patients could improve the detection of outbreaks caused by virulent STEC strains. Focussing on paediatric HUS patients is likely to suffice since most cases are children. In the past, HUS surveillance studies were time-limited and focussed primarily on clinical and microbiological characteristics of the infection. In contrast, the aspect of identifying STEC outbreaks through HUS surveillance has received only little attention. In fact, it may seem counterintuitive to use a post-infectious syndrome as an outbreak indicator because signal detection is clearly delayed (HUS commences about a week after onset of diarrhoea and the time elapsed between exposure and development of HUS is about two weeks). But, as outlined, outbreaks of non-0157:H7 and probably even 0157 infections are easily missed in current STEC surveillance. Additional systematic and prompt data collection of HUS patients in a surveillance framework may partially compensate for difficulties in current STEC surveillance schemes. This would also make the most of the extensive microbiological and epidemiological investigation that is often routinely conducted in individual HUS patients.

\section{References}

1. De Schrijver K, Buvens G, Possse B, Van den Branden D, Oosterlynck C, De Zutter $\mathrm{L}$, et al. Outbreak of veroytotoxin-producing E.coli 0145 and 026 infections associated with the consumption of ice cream produced at a farm, Belgium, 2007. Euro Surveill. 2008;13(7). Available from: http://www.eurosurveillance. org/edition/v13n07/080214_5.asp

2. Gerber A, Karch H, Allerberger F, Verweyen HM, Zimmerhackl LB. Clinical course and the role of shiga toxin-producing Escherichia coli infection in the hemolytic-uremic syndrome in pediatric patients, 1997-2000, in Germany and Austria: a prospective study. J Infect Dis. 2002;186(4):493-500.

3. Community outbreak of hemolytic uremic syndrome attributable to Escherichia coli 0111:NM--South Australia 1995. MMWR Morb Mortal Wkly Rep. 1995; 44(29):550-1,557-8. Available from: http://www.cdc.gov/mmwr/preview/ mmwrhtml/00038232.htm

4. Schimmer B, Eriksen HM, Nygård K, Grahek-Ogden D, Madssen T, Hajdu A, et al. An outbreak of haemolytic uraemic syndrome associated with minced beef, Norway, January-February 2006: preliminary report. Euro Surveill. 2006;11(3):E060302.1. Available from: http://www.eurosurveillance.org/ ew/2006/060302.asp

5. Eurosurveillance Editorial team. E. coli 0157 infections in the UK. Euro Surveill. 2006;11(6):E060601.2. Available from: http://www.eurosurveillance. org/ew/2006/060601.asp\#2

This article was published on 28 February 2008

Citation style for this article: Looking for tips to find icebergs - surveillance of haemolytic uraemic syndrome to detect outbreaks of Shiga toxin-producing $E$. coli infection. Euro Surveill. 2008;13(9):pii=8053. Available online: http://www. eurosurveillance.org/ViewArticle.aspx?ArticleId $=8053$ 